\title{
Coarse Integral Volumetric Imaging with Flat Screen and Wide Viewing Angle
}

\author{
Shimpei Sawada* and Hideki Kakeya \\ University of Tsukuba \\ 1-1-1 Tennoudai, Tsukuba 305-8573, JAPAN
}

\begin{abstract}
Coarse integral volumetric imaging (CIVI) combines multiview and volumetric display solutions and presents undistorted floating 3D image by correcting distortion of volumetric image for each view. In the conventional CIVI with limited viewing angle, distortions of image planes can be approximated to be parabolic in the direction of depth, while those in horizontal and vertical directions can be ignored. When the viewing angle becomes wider, however, this approximation cannot realize presentation of undistorted image. To cope with the strong distortions, the method the authors propose calculate z-coordinate of the generated real image is in detail and depicts each pixel on the display panel of the corresponding depth. Also distortions in horizontal and vertical directions are corrected by using texture mapping. To attain precise correction in vertical, horizontal and depth directions, optical paths of light rays between the display panel and each viewpoint are calculated with an optical simulator. Color aberration can also be corrected by mapping red, green and blue textures separately based on the result of the optical simulation.
\end{abstract}

kyewords

coarse integral volumetric imaging, volumetric display, multiview display, real image, aberration, distortion, viewing angle, lens

\section{INTRODUCTION}

Integral imaging, which combines fly-eye lenses with a high resolution flat display panel, is a prominent 3D display system in the sense that it can show not only horizontal parallax but also vertical parallax [1]. In the conventional integral imaging, the number of pixels each elemental lens of the fly-eye lens sheet covers is usually the same as the number of views, which means that the viewer perceives each elemental lens as one pixel. Therefore the focus of the viewer's eyes is always fixed on the screen (fly-eye lens sheet), which makes it hard to show realistic images far beyond the screen or popping up from the screen.

Besides the orthodox integral imaging described above, we can also think of integral imaging where each elemental lens is large enough to cover pixels dozens of times more than the number of views[2,3,4]. Kakeya defined this type of integral imaging as coarse integral imaging (CII) [5]. The advantage of CII is that it can induce focal accommodation off the screen, for it generates a real image or a virtual image with the lenses. Thus we can show realistic images far beyond the screen or popping up from the screen. Yet it cannot overcome the problem of vergence-accommodation conflict because the eyes of the viewer are always focusing on the real image or the virtual image generated by the lens array.

To solve this problem, Kakeya et al. proposed coarse integral volumetric imaging (CIVI), which combines volumetric solution with the multiview solution based on CII [5,6,7]. To realize CIVI, layered transparent display panels are used instead of a single layer display panel in CII. When multi-layered display panels are used, we can show volumetric real image or virtual image. To express pixels between image planes we can apply DFD approach[8,9,10], where 3D pixels are expressed with two adjacent panels, each of which emits light in inverse proportion to the distance between the 3D pixels and the panel. With this method we can overcome the shortcomings of multiview displays and volumetric displays at the same time. Conventional volumetric displays can achieve natural 3D vision without contradiction between binocular convergence and focal accommodation, while they cannot express occlusion or gloss of the objects in the scene. On the contrary multiview displays can express the latter while it cannot achieve the former. CIVI display can realize both natural 3D vision and expression of occlusion and gloss of the objects at the same time.

*E-mail : e0711210@edu.esys.tsukuba.ac.jp Phone\&FAX : +81-29-853-5381

Stereoscopic Displays and Applications XXII, edited by Andrew J. Woods, Nicolas S. Holliman,

Neil A. Dodgson, Proceedings of SPIE-IS\&T Electronic Imaging, SPIE Vol. 7863, 78631L

(C) 2011 SPIE-IS\&T · CCC code: 0277-786X/11/\$18 - doi: 10.1117/12.872287 
Though CIVI has solved most of the major problems of conventional 3D displays, it still has shortcomings to be overcome. One of the major problems left to be solved for CIVI is the limited viewing angle because of the aberration caused by the lenses. One solution to expand the viewing angle of CIVI display is to put together small CIVI display components, each of which has little aberration. Though aberration is reduced with this method, it still has a couple of problems to be overcome. One is the distinct seam between the small CIVI units, which can disturb the observer and decrease reality of the presented image. The other is that the screen of the display becomes curved and requires large space for placement.

In this paper we propose new distortion correction scheme that enables flat screen CIVI with wide viewing angle and little distortion. This paper is organized as follows. In section 2 principle of CIVI is reviewed. In section 3 a new distortion correction scheme is proposed. In section 4 experimental results of the proposed method is discussed. In section 5 summary of the paper is given.

\section{COARSE INTEGRAL VOLUMETRIC IMAGING}

Coarse integral imaging (CII) is a type of integral imaging where coarse fly-eye lens sheet (convex lens array) is used instead of fine fly-eye lens, which is usually used for integral imaging. In the conventional integral imaging, the number of pixels each elemental lens of the fly-eye lens sheet covers is usually the same as the number of views. The viewer perceives each elemental lens as one pixel and the focus of the viewer's eyes is always fixed on the screen. In CII, however, each elemental lens covers dozens by dozens or hundreds by hundreds pixels. The observable number of pixels through each lens is equal to the number of pixels covered by each lens divided by the square of magnifying power of the optical system. For example, when 100X100 pixels are covered by each lens of the fly-eye lens sheet and the magnifying power given by the pair of lenses (elemental lens and large aperture lens) is 10, 100 pixels are observed through each elemental lens.

With CII we can express 3D space far beyond the screen or popping up from the screen. To present 3D space far beyond the screen we can use virtual image, which is generated when the distance between the display panel and the lens sheet is nearer than the focal length of the elemental lenses of the lens sheet. We can express 3D objects popping up from the screen by generating real image, which emerges when the distance between the display panel and the lens sheet is farther than the focal length of the elemental lenses of the lens sheet. In the actual real-image CII we usually use a large aperture Fresnel lens in addition to the fly-eye lens sheet to converge light, for the aberration becomes small with this configuration. The distance between the display panel and the lens array is kept almost the same as the focal distance of the elemental lenses of the lens array so that the light may be collimated by each elemental lens. Then the large aperture convex Fresnel lens converges the collimated light and generates a real image at the focal distance of the Fresnel lens away from its surface.

The distance between the large aperture convex lens and the fly-eye lens sheet can vary. When the distance is short, the optics is similar to that of the traditional integral imaging and the total system size can be kept compact, while it can be a multiview system where the whole image observed in each eye switches alternately when we keep the distance between the lens array and the large aperture Fresnel lens long enough to generate the real image of the lens array, which corresponds to the viewing zone where the view for each eye changes alternately[11].

Though CII can show images off the screen, the problem of vergence-accommodation conflict still exists, for it can only generate one image plane. Besides the vergence-accommodation conflict, CII has another major problem that can severely damage the quality of image. When the distance between the lens array and the large aperture Fresnel lens is not far enough, multiple images from different elemental lenses are observed at the same time. In this case discontinuity of the images from different lenses becomes severe when the 3D image to be shown has large depth. To show depth of the image, CII depends only on the parallax of multiview images. The parallax among the images from different lenses has to be larger as the depth of the 3D object to be shown becomes longer. Consequently discontinuity of the images on the boundaries of the lenses ruins the quality of image as shown in Figure1.

One way to solve the problem of vergence-accommodation conflict and discontinuity of image at the same time is to introduce volumetric approach using multilayered panels in addition to the multiview approach $[12,13,14,15]$. In the scheme of coarse integral imaging, Kakeya et al. proposed coarse integral volumetric imaging (CIVI) as shown in Figure 2. In CIVI multiple display panels are inserted to generate volumetric real image (volumetric virtual image can also be generated if the display panels and the lens array are set closer) to keep the parallax between the images from two 
adjacent lenses small enough. Since artificial parallax is kept small, discontinuity between images from adjacent lenses are also kept small. Vergence-accommodation conflict is also reduced since each 3D pixel is displayed at the real image (virtual image) layer near the right depth. To express pixels between two panels we can use DFD approach, where 3D pixels are expressed with two adjacent panels, each of which has the pixel intensity in inverse proportion to the distance between the 3D pixels and the panel. Thus natural continuity of depth is realized.

Here it should be noted that the layered real images generated by the lenses are curved and distorted. Not only the generated image plane is distorted, but also the image planes generated by elemental lenses of the lens array are not uniform. The image planes generated by the elemental lenses off the optical axis of the large aperture Fresnel lens do not have line symmetry about the optical axis, but are slanted toward the optical axis. The slant becomes greater as the elemental lens goes farther from the optical axis. Without taking into account this distortion problem, smooth connection of elemental images cannot be realized. To cope with these distortions we can apply DFD for distorted image plane, which can realize natural connections between elemental images. Figure 3 shows the prototype system of the coarse integral volumetric display and an example of the 3D still picture shown with the prototype system.

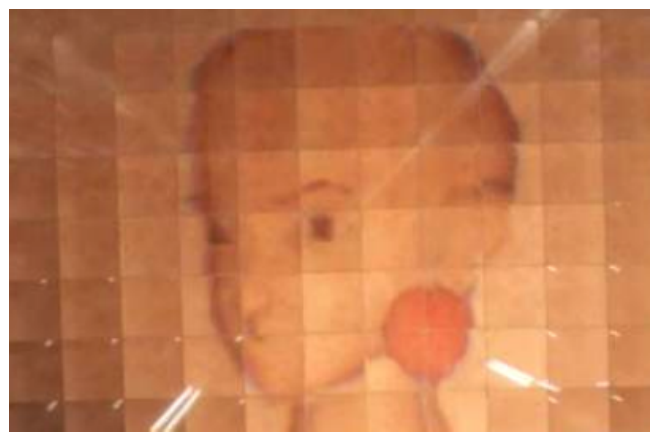

Figure 1: Discontinuity of image presented by CII display.

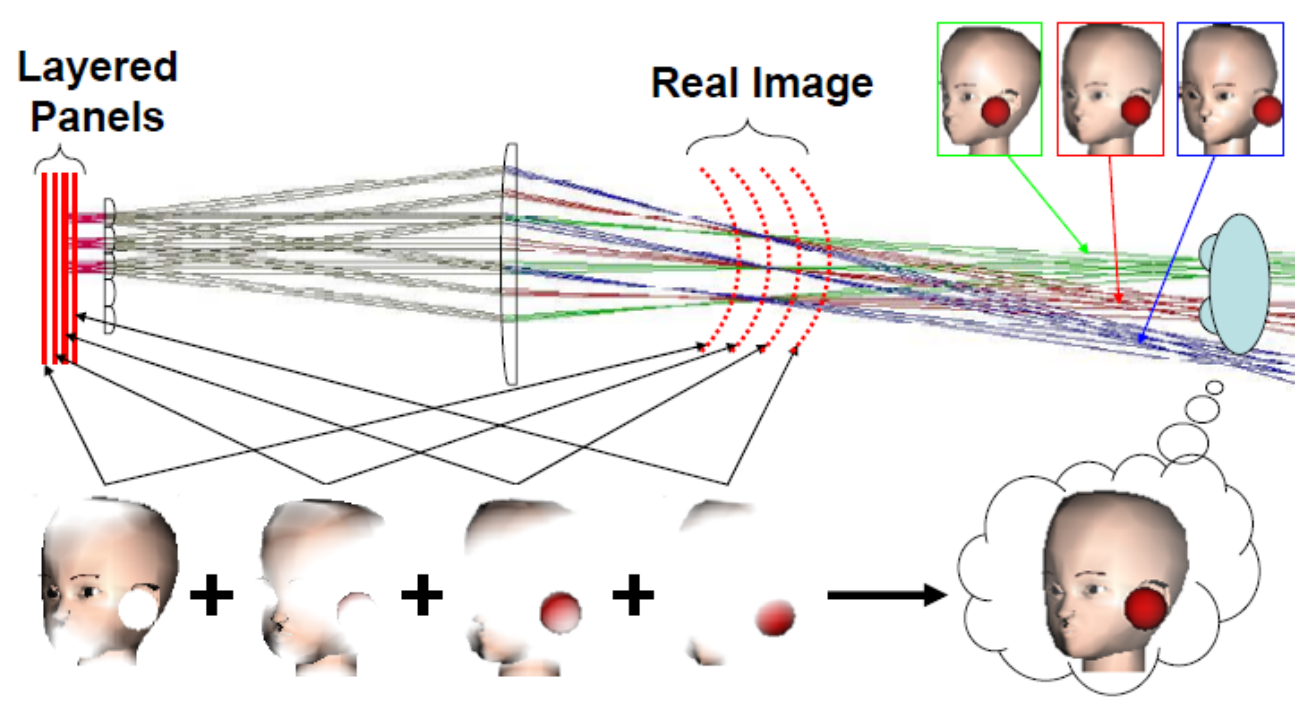

Figure 2: Principle of coarse integral volumetric imaging. 

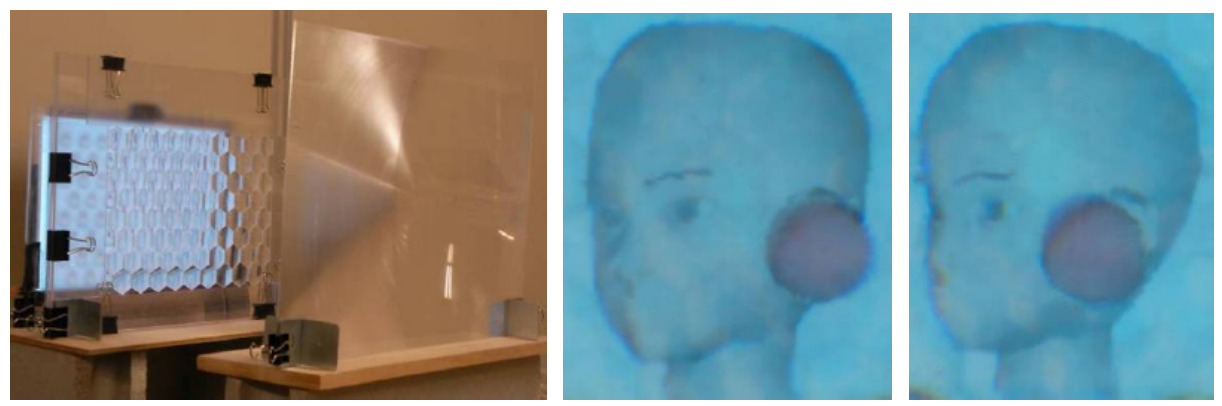

Figure 3: Prototype of coarse integral volumetric imaging (left) and the images observed from two different viewpoints with the prototype system (middle and right).

Now we give a detailed explanation on how the layered real images generated by the lenses are curved and distorted. When the viewing angle of floating 3D image is limited, distortion of real image planes generated by the lenses of CIVI can be approximated to be parabolic. The parameters of the parabolic function can be obtained based on the result of the optical simulation. By calculating refraction of light rays from the points on the display panel, we can obtain the points where the light rays converge, which are defined as the points on the real image. Then we can obtain the best-fit parabolic curve for these points by the minimum square error method. The axis of the parabolic function of the distorted image observed from each viewpoint is approximated to be on the line that connects the viewpoint to the focal point of the large aperture lens. The distortion in horizontal and vertical directions is not distinct and is negligible in the central part of the elemental images. Therefore we only need to apply DFD for distorted image planes for each elemental image by calculating the distance between each image plane and the $3 \mathrm{D}$ pixel to be presented.

Though this approximation is simple and requires little calculation for correction of distortion, it cannot be applied to CIVI with large viewing angles, for the way of distortion becomes stronger and more complex. In the next section we propose a new method of distortion correction to cope with this problem.

\section{CORRECTION OF STRONG DISTORTION}

When the viewing angle becomes wider, we have two problems to overcome. The first problem is that the way of distortion in the depth direction cannot be approximated by a simple parabolic function [16]. The second problem is that distortion in horizontal and vertical directions becomes distinct and has to be corrected to show undistorted image. To solve these problems, this paper proposes a new scheme of distortion correction. As for the correction of distortion in horizontal and vertical directions, we trace light rays from the viewpoint to the display panel and calculate how the images are distorted in those directions. Then texture mapping is used to correct distortion based on the result of optical simulation $[17,18]$. As for the correction of distortion in the direction of depth, we calculate refraction of light rays from the points on the display panel and obtain the points where the light rays converge.

The basic idea of the method we propose here is to show the image distorted in the reverse direction on the display panel to present undistorted image to the viewer. Therefore this method can also be applied to cancel out chromatic aberration. If we apply the same calculation described above for red, green, and blue color components independently, chromatic aberration can be corrected.

Now we give a detailed explanation on how the distortion correction proceeds. First the image from each viewpoint is generated by the standard computer graphic technique. Here we assume that orthogonal projection is applied to generate a 2D image with depth values from a 3D space. Then we project the light rays that correspond to the orthogonal projection (parallel light) to the lenses on the optical simulator and calculate how the rays are refracted and which points on the display panel they reach after refraction. Figure 4 illustrates how this calculation proceeds.

Each point of the image generated by orthogonal projection should be displayed on the points of the display panel calculated and plotted by the above optical simulation. To realize it, we can use texture mapping technique as shown in Figure 5. By showing the image distorted in the opposite direction, undistorted image is expected to be observed by the viewer. 


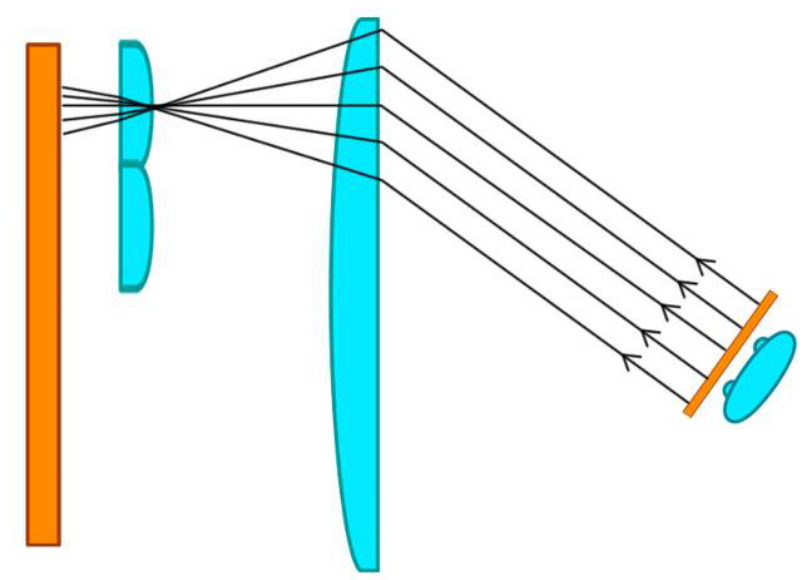

Figure 4: Illustration of how light rays coming from the orthogonal projection are refracted and reach the display panel
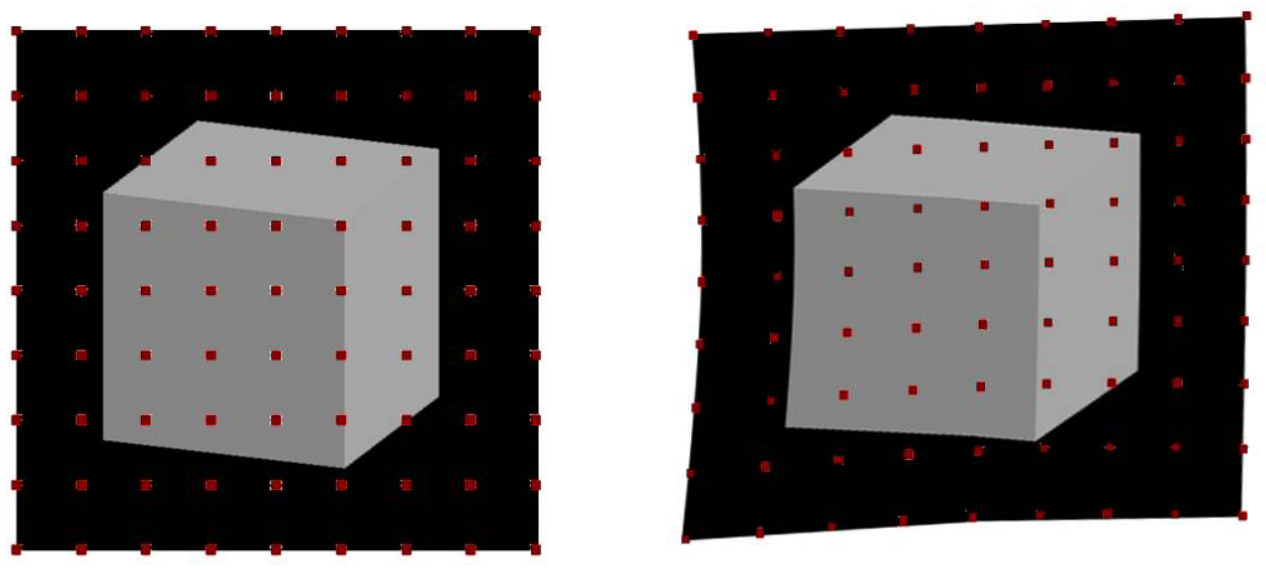

Figure 5: Example of an original image and the image to be display on the display panel.

Here it should be noted that we have to calculate and obtain the point arrays not only for one display panel, but also for the panels of all layers. The next step is to determine the layer on which each pixel is depicted, which requires information on where the real image of the elemental images on each layered panel is generated. Figure 6 illustrates how we can calculate the position and the shape of the generated real image. We calculate refraction of light rays from each point on the display panel obtained in the previous calculation for correction of distortion in horizontal and vertical directions, and obtain the points where the light rays converge.

Once the positions and the shapes of distorted image planes for each layer are obtained, we can generate the images to be displayed on each layer based on DFD for distorted image plane, where each 3D pixel is drawn on the adjacent two distorted image planes so that the pixel intensity may be in inverse proportion to the distance to each plane as shown in Figure 7. By applying this calculation for all the elemental images, we can realize presentation of undistorted 3D image even when the distortions given by the lenses are strong. 


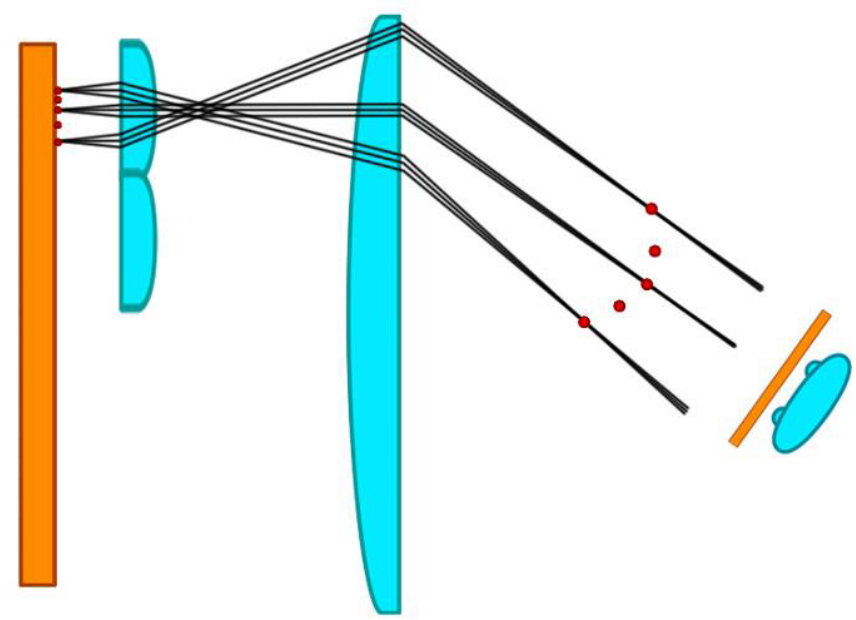

Figure 6: Calculation of position and shape of the real image generated by the lenses.

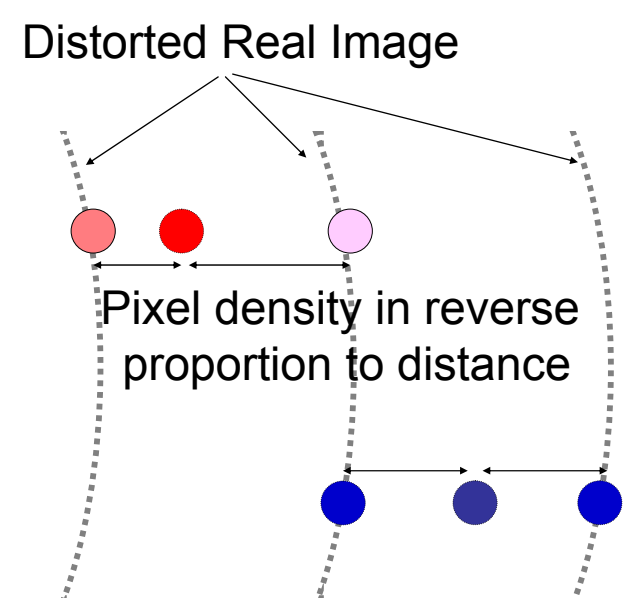

Figure 7: DFD for distorted image planes.

\section{EXPERIMENT AND RESULTS}

Based on the algorithm of strong distortion correction discussed in the previouse section, we made a CIVI system with 60 viewpoints. To realize a CIVI system with 3 layers, here we used half mirrors instead of layered panels to maintain brightness as shown in Figure 8. Detailed specifications of this prototype system are as follows:

- Resolution of display panels: 3840X2400 (Pixel Pitch: 0.1245mmX0.1245mm)

- Number of display layers: 3

- Number of views: 10X6

- Focal length of elemental lenses: $90 \mathrm{~mm}$ 
- Size of elemental lenses: $45 \mathrm{mmX} 45 \mathrm{~mm}$

- Distance between elemental lenses and display panels (3 layers): $89 \mathrm{~mm}, 90 \mathrm{~mm}, 91 \mathrm{~mm}$

- Distance between elemental lenses and large aperture lens: $970 \mathrm{~mm}$

- Focal length of large aperture lens: $600 \mathrm{~mm}$

- Size of large aperture lens: $500 \mathrm{mmX} 300 \mathrm{~mm}$
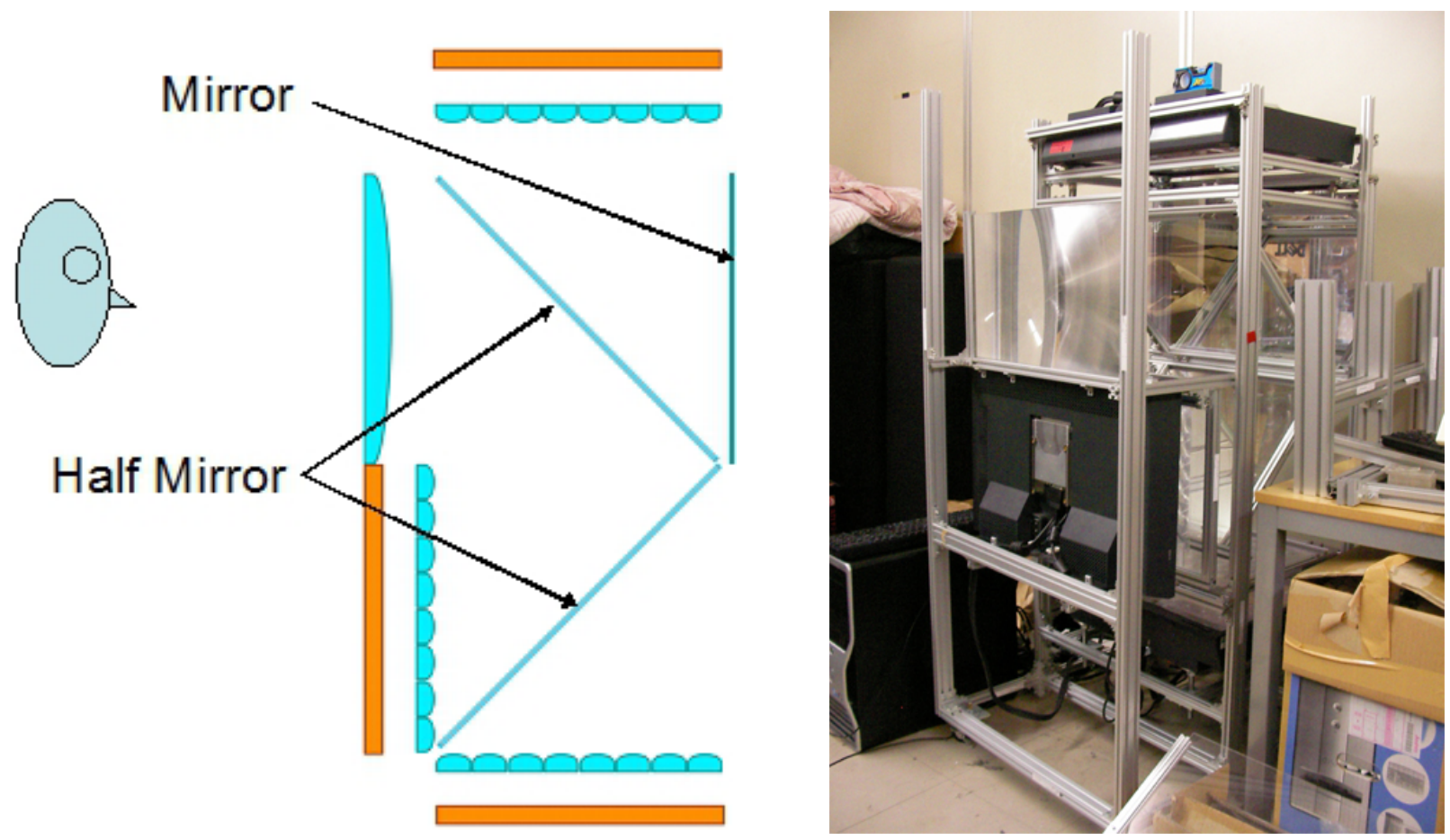

Figure 8: Prototype system of CIVI with wide viewing angle.

For this prototype system we applied the distortion correction algorithm introduced in the previous section. First we show the results of optical simulation using the optical parameters of the prototype system. Figure 9 shows how the distortion correction in horizontal and vertical diretions works in the prototype system. This figure shows distortion correction of the corner elemental image farthest from the optical center. Here 9X9 rays are traced and mapped. The left figure shows the points to be depicted on the display panel and the right figure shows how the points look like from the viewer. As shown in this figure, it is confirmed that the distortion correction works theoretically.

Figure 10 shows how the real image is distorted in this prototype. This figure again shows the distorted real image generated by the corner elemental lens farthest from the optical center. We can see the strong distortion in the direction of depth. We calculate distortion of the real images generated by all the elemental lenses and apply DFD for distorted image plane for each elemental image based on the result of these optical calculations.

Figure 11 shows the elemental images obtained by DFD for distorted image planes. Three images correspond to the images for three layers. The top, middle, and bottom pictures show the elemental images to be displayed on the front, middle, and back panels respectively. 

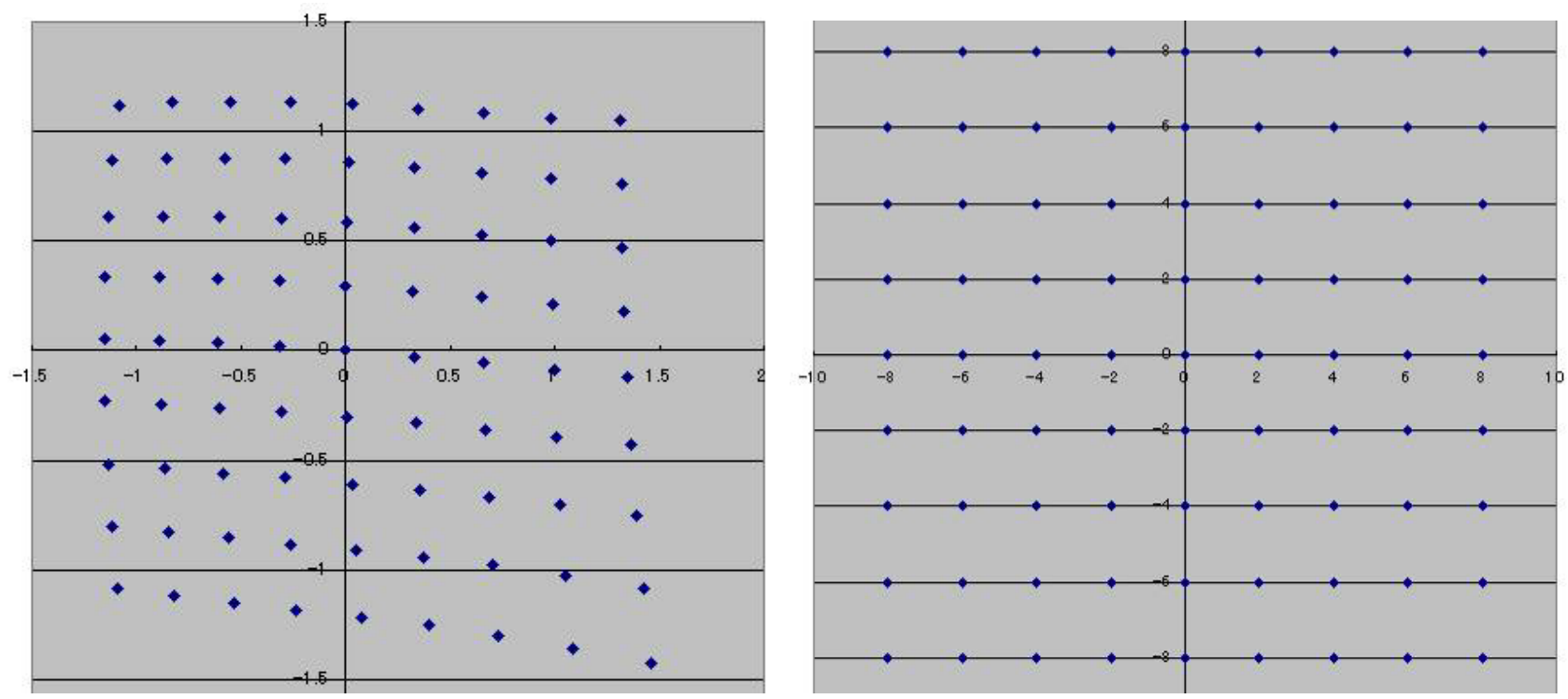

Figure 9: Distortion correction in horizontal and vertical directions.

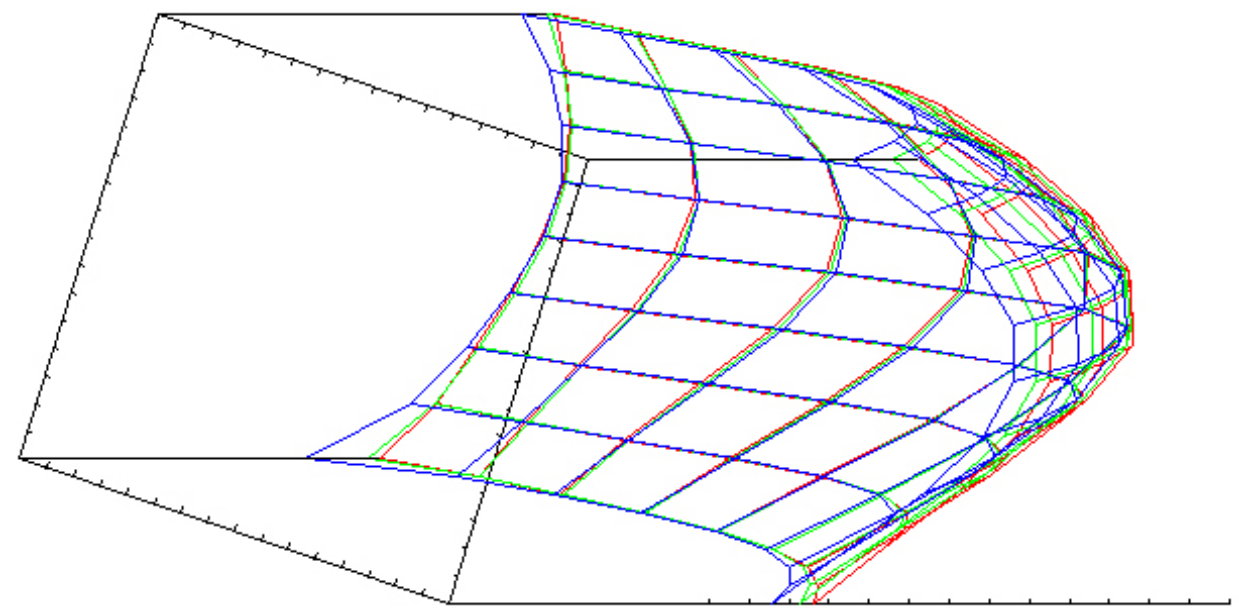

Figure 10: Distortion in depth direction.

Figures 12 and 13 pick up two of the 60 elemental images and show how they are observed with the prototype system. The original image (left) is divided into three layers according to the calculation of depth (center). Here note that the image color is also divided because the refraction rates of three color rays are different. From the viewpoint these three images are merged (right). Also distortion of image is corrected even when the view is observed from wide angles as shown in Figure 13. Thus it is confirmed that the proposed algorithm realizes a CIVI display with little distortion and wide viewing angle as expected. 


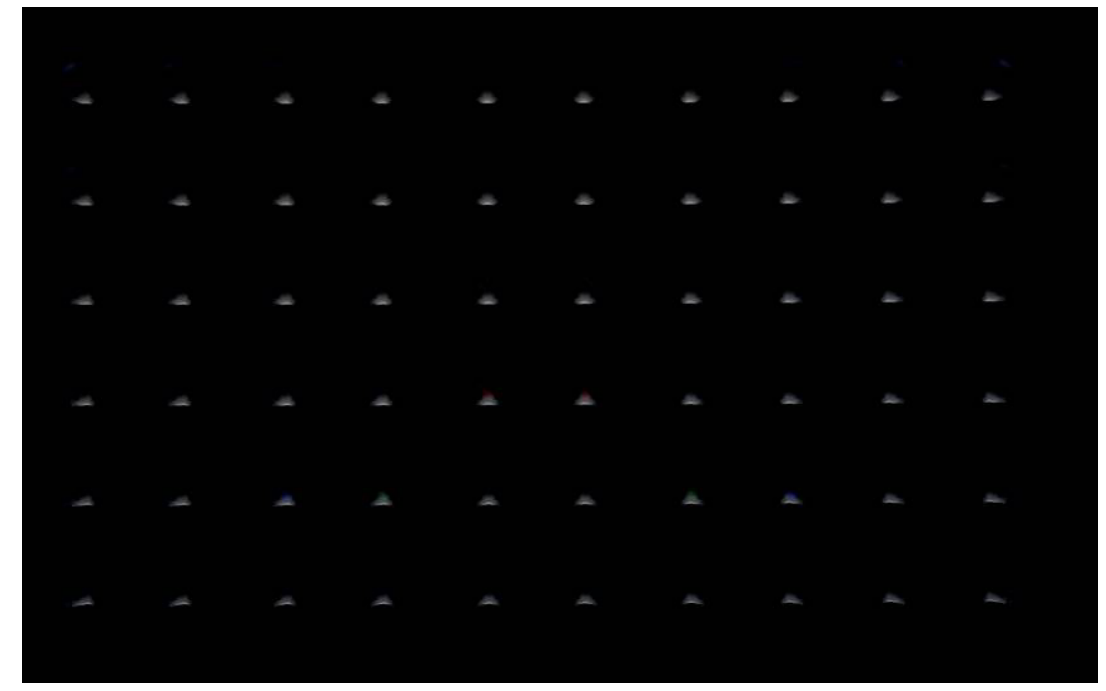

\begin{tabular}{|c|c|c|c|c|c|c|c|c|c|}
\hline 8 & 8 & 8 & 8 & 8 & 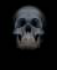 & 8 & 8 & 8 & 8 \\
\hline 8 & 6 & 8 & 28 & 8 & A & 8 & 28 & 2 & 8 \\
\hline 8 & 62 & 8 & 6 & 6 & $a$ & 28 & 28 & 2 & 2 \\
\hline 6 & 6 & 6 & 2 & 2 & 2 & 2 & 2 & 2 & 2 \\
\hline 6 & 6 & 62 & 2 & 2 & 88 & 20 & 28 & 28 & 2 \\
\hline 6 & 6 & 2 & 28 & 2 & 28 & 8 & 8 & 8 & 8 \\
\hline
\end{tabular}

\begin{tabular}{|c|c|c|c|c|c|c|c|c|c|}
\hline$A$ & 4 & 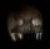 & $R R_{n}$ & 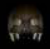 & 28 음 & 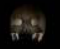 & 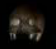 & $A$ & $A H$ \\
\hline A & 4 & $H$ & $2 \mathrm{Re}$ & $2 \mathrm{An}$ & 20 & $\pi *$ & 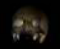 & F & A \\
\hline सa & 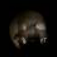 & 48 & $\mathrm{RS}$ & Ris & $\mathrm{AN}$ & ค요 & 애 & $A B$ & A \\
\hline A & 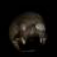 & $\mathrm{R}^{2}$ & Rat & Rat & $\mathrm{R}$ \% & 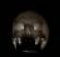 & 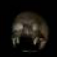 & 8 & As, \\
\hline$A$ & 8 & 8 & 82 & 28 & 용 & 28 & 98 & 8 & 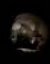 \\
\hline$A$ & 9 & 89 & $a_{3}$ & $(39)$ & (A2) & 98 & 89 & 9 & SP \\
\hline
\end{tabular}

Figure 11: Elemental images. 

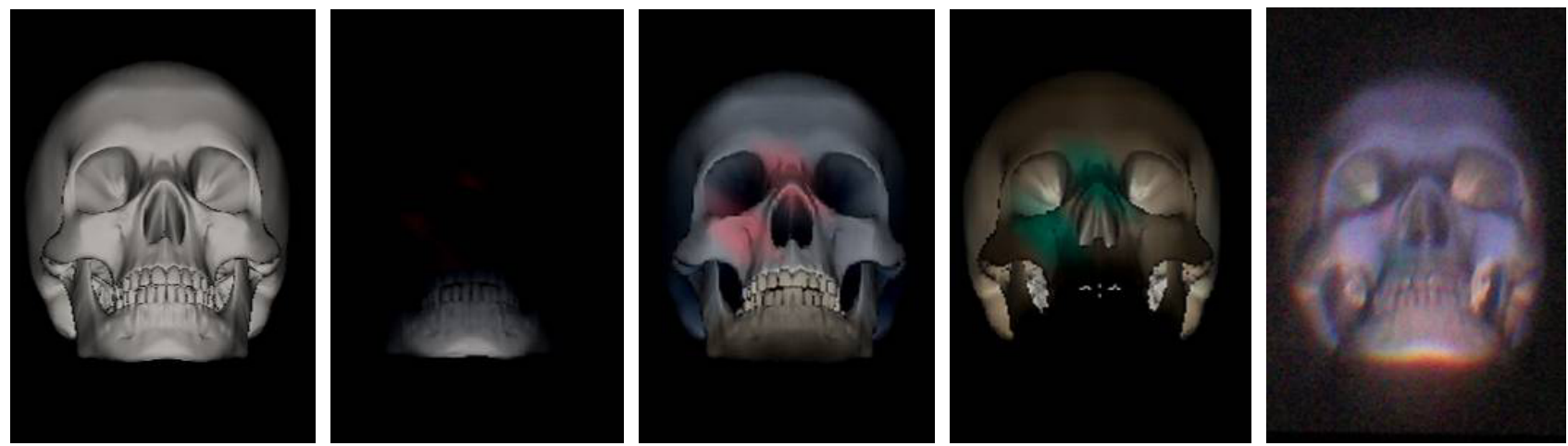

Figure 12: Original image, displayed images on each layer, and the image observed from the central viewpoint
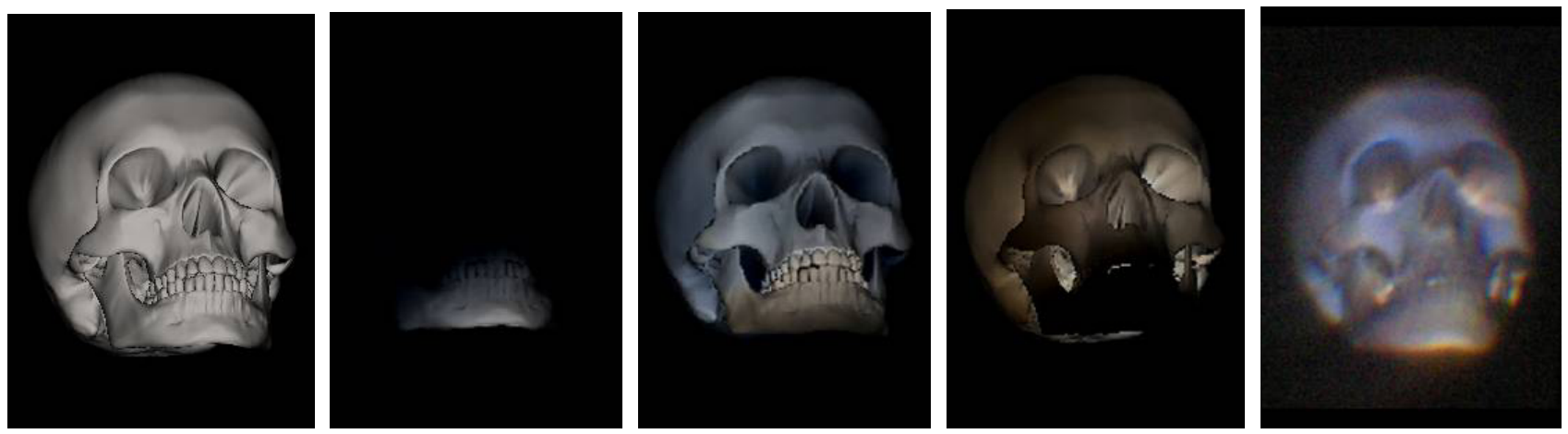

Figure 13: Original image, displayed images on each layer, and the image observed from the leftmost viewpoint.

\section{SUMMARY}

In the present paper, new distortion correction scheme is proposed to enable flat screen CIVI with wide viewing angle and little distortion. To cope with strong distortion that appears in CIVI with small $\mathrm{F}$ numbers, distortion is corrected in vertical, horizontal and depth directions by calculating the optical paths of light rays between the display panel and each viewpoint with an optical simulator. Distortions in depth direction are corrected by applying DFD for distorted image plane based on z-coordinate of the generated real images calculated in detail by the optical simulator. Distortions in horizontal and vertical directions are corrected by using texture mapping based on the result of the optical simulation also. By showing the image distorted in the reverse direction, undistorted image is observed by the viewer. Also color aberration is corrected by mapping red, green and blue textures separately based on the result of the optical simulation using different refraction rates. With the prototype system it is confirmed that the proposed method realizes a CIVI display with little distortion and wide viewing angle as expected.

\section{ACKNOWLEDGEMENT}

This research is partially supported by the Grant-in-Aid for Scientific Research, MEXT, Japan, Grant number: 22680008 . 


\section{REFERENCES}

[1] G. Lippmann, "La photograhie integrale," Comptes Rendus Acad. Sci. 146, 446-451 (1908).

[2] B. Lee, S. Jung, S.-W. Min, and J.-H. Park, "Three-dimensional display by use of integral photography with dynamically variable image planes," Opt. Lett. 26, 1481-1482 (2001).

[3] J.-H. Park, S. Jung, H. Choi, and B. Lee, "Integral imagingwith multiple image planes using a uniaxial crystal plate," Opt. Express. 11, 1862-1875 (2003).

[4] S.-W. Min, B. Javidi, and B. Lee, "Enhanced three-dimensional integral imaging system by use of double display devices,"Appl. Opt. 42, 4186-4195 (2003).

[5] H. Kakeya, "Coarse integral imaging and its applications," Proc. SPIE proceeding Volume 6803: Stereoscopic Displays and Virtual Reality Systems XV, 680317 (2008).

[6] H. Kakeya, "Improving Image Quality of Coarse Integral Volumetric Display," SPIE proceeding Volume 7237: Stereoscopic Displays and Virtual Reality Systems XVI, 723726 (2009).

[7] H. Kakeya, T. Kurokawa, Y. Mano, "Electronic Realization of Coarse Integral Volumetric Imaging with Wide Viewing Angle," SPIE proceeding, (2010)

[8] S. Suyama, H. Takada, K. Uehira, S. Sakaiand S. Ohtsuka, "A Novel Direct-Vision 3-D Display using Luminance-Modulated Two 2-D Images Displayed at Different Depths," SID’00 Digest of Technical Papers, 54.1, pp. 1208-1211, (2000).

[9] S. Suyama, H. Takada and S. Ohtsuka, "A Direct-Vision 3-D Display Using a New Depth-fusing Perceptual Phenomenon in 2-D Displays with Different Depths," IEICE Trans. on Electron., Vol. E85-C, No. 11, pp.1911-1915 (2002).

[10] S. Suyama, S. Ohtsuka, H. Takada, K. Uehira and S. Sakai, "Apparent 3-D image perceived from luminance-modulated two 2-D images displayed at different depths," Vision Research, 44, pp. 785-793 (2004).

[11] H. Kakeya, "MOEVision: simple multiview display with clear floating image," SPIE proceeding Volume 6490: Stereoscopic Displays and Virtual Reality Systems XIV, 64900J (2007)

[12] R. Yasui, I. Matsuda, H. Kakeya, "Combining volumetric edge display and multiview display for expression of natural 3D images," SPIE Proceedings Volume 6055 (2006).

[13] H. Ebisu, T. Kimura, H. Kakeya, "Realization of electronic 3D display combining multiview and volumentric solutions," SPIE Proceedings Volume 6490 (2007).

[14] Y. Kim, J.-H. Park, H. Choi, J. Kim, S.-W. Cho, and B. Lee, "Depth-enhanced three-dimensional integral imaging by use of multilayered display devices," Appl. Opt. 45, 4334-4343 (2006).

[15] Y. Kim, H. Choi, J. Kim, S. -W. Cho, Y, Kim, J. Park, and B. Lee, "Depth-enhanced integral imaging display system with electrically variable image planes using polymer-dispersed liquid-crystal layers," Appl. Opt. 46, 3766-3773 (2007).

[16] H. Kodaira, A. Nakao, Y. Mano and H. Kakeya, "Distortion correction of coarse integral volumetric imaging with large lens," Proc. Of 3DSA, pp. 205-208 (2010).

[17] H. Kakeya and Y. Arakawa, "Autostereoscopic Display with Real-image Virtual Screen and Light Filters," SPIE Proceedings Volume 4660 (2002).

[18] H. Kakeya, "Real-Image-Based Autostereoscopic Display Using LCD, Mirrors, and Lenses," SPIE Proceedings Volume 5006 (2003). 